\title{
Solar activity and responses observed in Balmer lines
}

\author{
S. Marchenko ${ }^{1,2}$, S. Criscuoli ${ }^{3}$, M. T. DeLand ${ }^{1,2}$, D. P. Choudhary ${ }^{4}$, and G. Kopp ${ }^{5}$ \\ 1 Science Systems and Applications, Inc., Lanham, MD, USA \\ e-mail: sergey.marchenko@ssaihq.com \\ 2 Goddard Space Flight Center, Greenbelt, MD, USA \\ 3 National Solar Observatory, Boulder, CO, USA \\ ${ }^{4}$ San Fernando Observatory, California State University, Northridge, CA, USA \\ ${ }^{5}$ University of Colorado/LASP, Boulder, CO, USA
}

Received 18 February 2020 / Accepted 12 December 2020

\begin{abstract}
Context. Many stars show Sun-like magnetic activity cycles, which are frequently observed by tracking changes in the chromospherically sensitive CaII H\&K doublet. However, relationships between the line profile changes related to the magnetic activity seen in strong spectral transitions in other portions of a stellar spectrum are yet to be understood.

Aims. We follow variability patterns in various solar lines in order to relate them to the emergence, passage, and decay of active solar regions.

Methods. The line activity indices (core-to-wing ratio) for the upper Balmer lines - $\mathrm{H} \beta, \mathrm{H} \gamma$, and $\mathrm{H} \delta$ - are constructed from the near-daily solar measurements acquired by the Ozone Monitoring Instrument and the TROPOspheric Monitoring Instrument. Results. On solar rotation timescales, the upper Balmer line activity indices closely follow variations in the total solar irradiance, $r \sim-(0.6-0.7)$, and thus frequently deviate from the behavior of the line activity indices that track chromospheric activity levels (e.g., the $\mathrm{CH} 430 \mathrm{~nm}$ band used in this study), specifically during passages of big sunspot groups.
\end{abstract}

Key words. Sun: activity - Sun: atmosphere

\section{Introduction}

The search for exoplanets around Sun-like stars frequently calls for a detailed accounting of the intrinsic stellar activity, which is routinely assessed through changes in prominent spectral lines, CaII, NaI, and $\mathrm{H} \alpha$ in particular. Recent multi-year long-term surveys of sizeable samples of F-M (Cincunegui et al. 2007) and F-G-K stars (Gomes da Silva et al. 2014) provide a perplexingly wide variety of cases, from well-correlated to independent and even anticorrelated behavior of the CaII and $\mathrm{H} \alpha$ line fluxes (Cincunegui et al. 2007). For example, in two solar analogs, HD 45184 (Flores et al. 2016) and HD 38858 (Flores et al. 2018), the rising line-core fluxes (line-filling and shallowing in a typical reaction to a rising stellar activity) in the CaII H\&K doublet lead to a stronger (deeper) profile of the Balmer $\mathrm{H} \beta$ line.

Long-term observations show that the solar full-disk (Sunas-a-star) line activity indices of $\mathrm{H} \alpha$ track the CaII H\&K doublet on solar-cycle timescales, albeit with much lower relative amplitude (Livingston et al. 2007, 2010). Using the data from Livingston et al. (2007), Meunier \& Delfosse (2009) found that the correlation between $\mathrm{CaII}$ and $\mathrm{H} \alpha$ varies throughout the solar cycle, strengthening around solar maximum and weakening at solar minimum, and displays asymmetric behavior at the ascending and descending solar-cycle branches. This complex relationship was ascribed to the different sensitivity of lines to the fluxes arising from plages and dark filaments.

The simultaneous long-term measurements of the total and spectral solar irradiances (TSI and SSI, respectively) by SORCE (SOlar Radiation and Climate Experiment) show that the Lyman- $\alpha$ variability patterns deviate from the TSI changes on
Carrington rotational timescales, especially during the highactivity epochs when the line frequently anticorrelates with TSI. The $\mathrm{H} \alpha$ line-flux, though, mostly follows TSI (Lee et al. 2016).

In this paper we focus on Balmer lines, whose observations serve several purposes in stellar astrophysics. Balmer lines are excellent tracers of the stellar effective temperature (Fuhrmann et al. 1994; Barklem et al. 2000; Amarsi et al. 2018). The lines have been used to constrain photospheric (Gardiner et al. 1999; Pereira et al. 2013), chromospheric (Dupree et al. 2016; Linsky 2017), and flare models (Cram \& Woods 1982; Kowalski et al. 2017).

Perusing the near-daily solar observations supplied by the Ozone Monitoring Instrument (OMI) in the $265-500 \mathrm{~nm}$ spectral domain, Marchenko \& DeLand (2014) noted that practically all strong spectral lines and blends varied in unison in the disk-integrated solar spectra, closely following the gradual solar cycle changes seen in the MgII and CaII transitions: The lines grew progressively shallower with rising solar activity levels. The cores of upper Balmer lines of hydrogen $(\mathrm{H} \beta, \mathrm{H} \gamma$, and $\mathrm{H} \delta$ in that particular study) bucked the general trend, showing negligible line-filling effects on solar-cycle timescales. Moreover, the authors did not rule out the possibility of a gradual deepening of the Balmer line profiles along the developing solar cycle, linking the effect with the theoretical predictions by Przybilla \& Butler (2004). All documented effects, however, fell close to the estimated uncertainty thresholds, thus calling for an independent confirmation.

The recent study of the upper Balmer lines, among other transitions, by Maldonado et al. (2019) demonstrated that the solar full-disk line activity indices of $\mathrm{H} \beta, \mathrm{H} \gamma$, and $\mathrm{H} \delta$ 
Table 1. Line activity indices.

\begin{tabular}{lcc}
\hline \hline Line & Center $(\mathrm{nm})$ & Width $(\mathrm{nm})$ \\
\hline $\mathrm{H} \beta$ red wing & 488.0 & 0.5 \\
$\mathrm{H} \beta$ core & 486.22 & 0.3 \\
$\mathrm{H} \beta$ blue wing & 484.95 & 0.7 \\
$\mathrm{H} \gamma$ red wing & 436.55 & 0.7 \\
$\mathrm{H} \gamma$ core & 434.07 & 0.3 \\
$\mathrm{H} \gamma$ blue wing & 433.35 & 0.3 \\
$\mathrm{H} \delta$ red wing & 412.45 & 0.5 \\
$\mathrm{H} \delta$ core & 410.22 & 0.3 \\
$\mathrm{H} \delta$ blue wing & 409.0 & 0.4 \\
$\mathrm{CH} 430$ nm red wing & 432.05 & 0.3 \\
$\mathrm{CH} 430 \mathrm{~nm}$ core & 430.65 & 0.9 \\
$\mathrm{CH} 430 \mathrm{~nm}$ blue wing & 428.2 & 0.8 \\
\hline
\end{tabular}

anticorrelate with the CaII values, though with diminishing significance of the correlation coefficients (much more negative for $\mathrm{H} \beta$ than $\mathrm{H} \delta$ ). The $\mathrm{H} \alpha$ indices showed higher noise, mostly following the rest of the upper Balmer lines. The $\mathrm{H} \alpha$-CaII anticorrelation was somewhat lessened during periods of high solar activity. $\mathrm{H} \epsilon$ reverted to following CaII.

Abundant high-resolution, high $\mathrm{S} / \mathrm{N}$ disk-integrated spectra taken over two solar rotational periods in June-August 2016 (Thompson et al. 2020) were used to construct a low-activity (no spots, very few faculae) spectral template. The relative (highactivity vs. low-activity) spectra reveal weak pseudo-emission and pseudo-absorption features associated with metal lines (Fe, $\mathrm{Ti}, \mathrm{Mn})$. These features correlate well with the activity indicators ( $\log R_{\mathrm{HK}}^{\prime}$, facula-filling factors), though much less so with the spot-filling factors and the line activity indices (upper Balmer lines, NaI, and HeI) from Maldonado et al. (2019).

Here we investigate the behavior of $\mathrm{H} \beta, \mathrm{H} \gamma$, and $\mathrm{H} \delta$ lines and show that they closely follow changes in the TSI. To do so, we combine the long-term record of the near-daily OMI solar observations with the near-daily spectral irradiance measurements from the recently launched TROPOspheric Monitoring Instrument (TROPOMI) mission.

\section{Data}

OMI (Levelt et al. 2006, 2018) has been operating on the Earth-observing Aura satellite since July 2004, providing longterm records of the atmospheric trace gases. The primary OMI measurement of backscattered terrestrial radiance requires frequent and reliable calibration, which is realized by observing the Sun at every 15 th orbit (more or less daily). These measurements are collected using a volume diffuser, providing "Sun-as-a-star" observations of solar activity. The spectrometer covers the $264-504 \mathrm{~nm}$ range with $0.4-0.6 \mathrm{~nm}$ resolution, acquiring spectra at 30-60 simultaneous (push-broom optical design) fields of view (FOVs). These multiple, simultaneously obtained disk-integrated solar spectra are sampled at slightly different wavelength grids, thus facilitating the construction of an oversampled, high signal-to-noise, $S / N>2000$, near-daily solar spectrum with $0.02 \mathrm{~nm}$ wavelength sampling steps.

The recently launched (October 2017) TROPOMI (Veefkind et al. 2012) generally follows the design of the predecessor OMI instrument, sampling the Earth's atmosphere in the 270$2385 \mathrm{~nm}$ range (270-495 $\mathrm{nm}$ contiguous coverage) with $0.2-$ $0.5 \mathrm{~nm}$ spectral resolution at each of the 450 simultaneously acquired FOVs. As with OMI, the near-daily 15th-orbit solar observations are performed through a volume diffuser (i.e., providing a Sun-as-a-star outlook). The greatly increased number of FOVs compared to OMI and the slightly shifted wavelength grids allow us to construct an over-sampled near-daily solar spectrum with $S / N \geq 10^{4}$.

We used the daily solar OMI and TROPOMI spectral irradiance data to construct the line activity indices by evaluating, for each given line, the ratios of the line-core fluxes to the wingaveraged fluxes, as specified in Table 1, which provides the central wavelengths and widths of the regions used to evaluate the indices. Though the indices are not as straightforward to interpret as line fluxes, their differential nature (the line core vs. the adjacent line wings) helps to lessen the impact of instrument changes. As we show below, the combination of excellent (for a space-borne spectrograph) long-term instrument stability (OMI: Schenkeveld et al. 2017; so far, TROPOMI follows the OMI lead), the simultaneous multi-FOV spectral acquisition, and the activity index approach leads to unprecedented low noise levels: $<500$ parts-per-million ( $\mathrm{ppm}$ ) for OMI and $<50 \mathrm{ppm}$ for TROPOMI (see below). These noise levels can be compared to the typical $\sim 3000 \mathrm{ppm}$ activity-index uncertainties $(1 \sigma)$ quoted for various space missions ${ }^{1}$, as well as the $1900 \mathrm{ppm}$ standard deviation in the CaII H\&K activity-index measurements in Maldonado et al. (2019).

Though we calculated the line activity indices for dozens of transitions and blends, here we focus on the behavior of $\mathrm{H} \beta, \mathrm{H} \gamma$, and $\mathrm{H} \delta$ lines, comparing them to the $\mathrm{CH} 430 \mathrm{~nm}$ indices that consistently show excellent correlation with the traditional chromospheric activity proxies, $\mathrm{MgII} h \& k$ and $\mathrm{CaII} \mathrm{H} \& \mathrm{~K}$. The individual indices are normalized to the solar-minimum average (for our purposes, chosen between April 2007 and August 2009) in the OMI case and to the low-activity period (October 28, 2018, to January 16,2019 ) for the TROPOMI data. The normalized $\mathrm{H} \beta, \mathrm{H} \gamma$, and $\mathrm{H} \delta$ indices are de-trended by subtracting 61-day running means and are then averaged. Combining $\mathrm{H} \beta, \mathrm{H} \gamma$, and $\mathrm{H} \delta$, we note that only the core of $\mathrm{H} \beta$ has non-negligible contribution from chromospheric layers (Avrett \& Loeser 2008). Before deciding to average these three lines, we carefully inspected the individual variability patterns, finding them to be similar to those within the noise. Hence, the (mostly instrument-related) limitations of the adopted approach do not allow us to detect the differences (if any) between the variability patterns arising from the predominantly photospheric-formed and the photospheric-andchromospheric-formed line cores.

A similar approach was applied to the $\mathrm{CH} 430 \mathrm{~nm}$ indices and the SORCE/TIM (Total Irradiance Monitor: Kopp \& Lawrence 2005) and TSIS-1/TIM (Total and Spectral Solar Irradiance Sensor: Pilewskie et al. 2018) TSI measurements, and we ultimately averaged the overlapping TSIs. We note that TSI is used here as a quantitative indicator of primarily sunspotdriven short-term activity changes, although facular (chromospheric) contributions are also present. The latter usually prevails on long-term (multi-month to decadal) timescales.

\section{Results}

We show the measured OMI and TROPOMI indices in Figs. 1 and 2. Choosing the relatively inactive epochs in the TSI records, we estimated the upper-limit noise levels (as defined, these include some, though relatively minor, components of the intrinsic solar variability) for the Balmer line activity indices as the

1 https://wWW.iup.uni-bremen.de/gome/gomemgii.html 


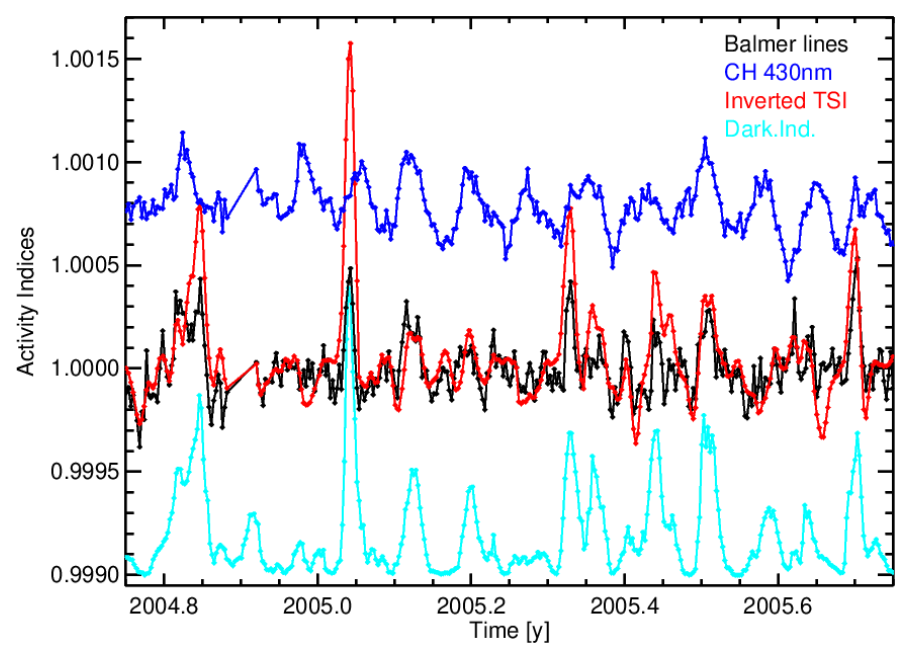

Fig. 1. Average de-trended Balmer activity indices derived from the near-daily OMI observations (black line) normalized to a minimum activity level and compared with the normalized and inverted TSI (red line), showing a clear correlation between the Balmer-line and TSI variability. For a reference, the OMI CH430nm activity indices (blue line) are normalized, downscaled, and shifted for clarity. The cyan line follows the NRLSSI2 sunspot darkening factors (Coddington et al. 2016), which are scaled and shifted for the sake of clarity. The Balmer line activity indices shown carry $7.8 * 10^{-5} 1-\sigma$ uncertainties.

rms (root mean square) of the corresponding Balmer indices: $78 \mathrm{ppm}$ for the OMI indices shown in Fig. 1, gradually growing to $304 \mathrm{ppm}$ in the epoch of simultaneous OMI and TROPOMI observations. TROPOMI delivers very high quality indices, with rms 32 ppm. Though aging "gracefully" (Schenkeveld et al. 2017), OMI shows gradually increasing noise levels in various products over the long mission time. This renders the latemission OMI line activity indices unsuitable for our purposes. Here we concentrate on the high-quality early-mission OMI data taken at the egress from the peak of solar cycle 23. However, we note that the behavior of the Balmer line activity indices shown in Fig. 1 persists through all of cycle 24: The most notable Balmer line changes (amidst the growing noise) are centered on the downward changes of TSI. TROPOMI data sample the minimum between cycles 24 and 25 with much lower activity levels and sparse, isolated active regions. Nevertheless, in Fig. 2, we specifically choose the event of emergence and rapid decay $(\sim 3$ rotational cycles) of two relatively large sunspot groups. Both the OMI and TROPOMI Balmer indices shown in Figs. 1 and 2 vary in line with the TSI values: linear regression correlation $r=-0.68$ for OMI and $r=-0.62$ for TROPOMI.

We note that the TSI variations are inverted in Figs. 1 and. 2 to emphasize the relationship with the Balmer lines, while the regression analysis uses the data as observed (hence $r<0$ ). It is obvious, especially in the OMI sample, that the variability in the Balmer lines is more closely related to inverted TSI than the chromospheric activity depicted by the $\mathrm{CH} 430 \mathrm{~nm}$ index. One may note the frequent misalignment of the inverted TSI+Balmer and $\mathrm{CH} 430 \mathrm{~nm}$ maxima. For example, the single $\mathrm{CH} 430 \mathrm{~nm}$ maximum at $T=2005.35$ transforms into a double-peaked appearance in the inverted-TSI and Balmer indices. There are, however, also frequent mismatches between the Balmer and TSI amplitudes (but good time-wise coincidence nevertheless) around some sunspot-driven episodes (e.g., at $T=2005.04$ and $T=2005.33)$.

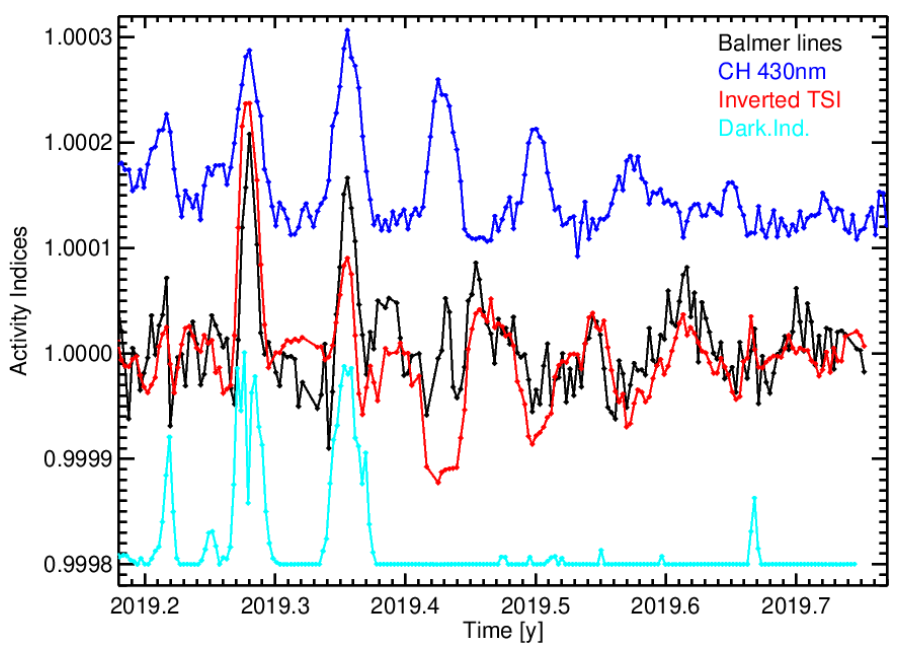

Fig. 2. Same as in Fig. 1, but for TROPOMI, again showing the correlation between the Balmer line activity indices (an upper limit point-topoint uncertainty: $\sigma=3.2 * 10^{-5}$ ) and TSI variability even in the near absence of sunspots.
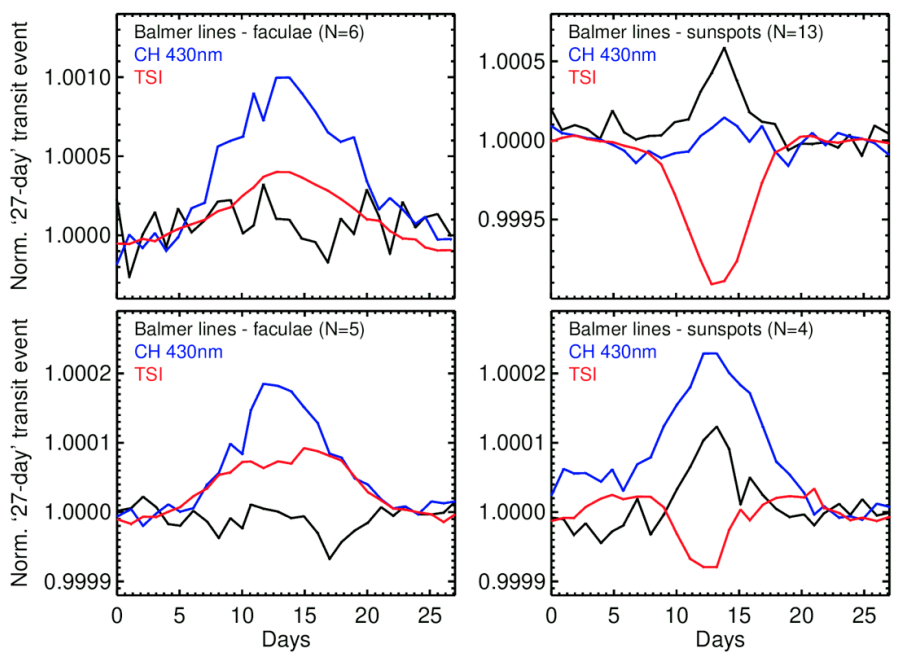

Fig. 3. Facula-dominated and sunspot-dominated events in the OMI and TROPOMI line activity indices. The upper panels show the OMI data: the facula and active network-dominating case (left panel) in comparison to the sunspot-dominating case (right panel). The lower panels show the same for TROPOMI. In all panels, the shown values are normalized to a low-activity epoch and averaged over multiple events $(N=4-13$ passages of active regions, as indicated in the upper-left corners of each panel). Black lines follow the Balmer activity indices, blue lines show the CH430nm indices, and red lines mark changes in TSI. We note the different $Y$ scales in the OMI and TROPOMI plots.

To compare the two driving factors, TSI and chromospheric activity, we segregated the available data into two categories: the sunspot-driven and the facular-flux-driven activity cycles. The former comprises the passages of prominent sunspot groups (downward TSI changes: $\triangle \mathrm{TSI} \geq 0.1 \%$ in the OMI record and $\Delta \mathrm{TSI} \geq 0.05 \%$ for TROPOMI). The latter combines the epochs of prominent $\mathrm{CH} 430 \mathrm{~nm}$ rotational modulation with either relatively low sunspot activity or with relatively small sunspot events clearly shifted from the CH430nm maxima (by $>0.25$ of the rotational cycle). Figure 3 shows the average profiles of these sunspot and facular events for both instruments.

The much higher solar activity level sampled by OMI leads to pronounced upward changes in the $\mathrm{CH} 430 \mathrm{~nm}$ indices (facular 
component) and the downward sunspot-related TSI variability. Despite the much lower activity levels in 2018-2019, the high S/N TROPOMI data reveal qualitatively similar trends: In the case of facular and active network flux driving TSI changes, the CH430nm index follows TSI, while the Balmer index remains practically flat, with a hint of the limb effects (the small dips at days $\sim 7-10$ and $\sim 15-20$ ). The sunspot TROPOMI case is similar to OMI, leading to a correlation of the Balmer index with the (inverted) TSI.

\section{Discussion}

Analysis of the disk-integrated near-daily OMI solar observations (Marchenko \& DeLand 2014) suggested that the upper Balmer lines deviate from the expected variability patterns (which have been observed on multiple occasions from space) on solar-cycle timescales (years). While the majority of the UV and visible transitions appeared to closely follow the chromospherically sensitive indicators (e.g., $\mathrm{Mg}$ II $h \& k$ and $\mathrm{Ca}$ II $\mathrm{H} \& \mathrm{~K}$ ), the observed $\mathrm{H} \beta, \mathrm{H} \gamma$, and $\mathrm{H} \delta$ lines were seemingly uninvolved (to within measurement errors) in the solar-cycle variability. A recent detailed study (Maldonado et al. 2019) backed this trend by demonstrating that on long-term scales (years), the disk-integrated line-activity indices of $\mathrm{H} \beta$ and $\mathrm{H} \gamma$ anticorrelate with $\mathrm{Ca}$ II $\mathrm{H} \& \mathrm{~K}$, while $\mathrm{H} \delta$ shows no statistically significant correlation.

The intricate upper Balmer variability patterns (Figs. 1 and 2) may explain the weak correlation between the pseudo-emission and pseudo-absorption features (centered on some Fe, Ti, and Mn lines) in the relative (thus sensitive to the rotational modulation) solar spectra (Thompson et al. 2020) and the Balmer line activity indices from Maldonado et al. (2019). The pseudoemissions are driven by changes in the facular surface-filling factors on solar rotational scales (Thompson et al. 2020). Hence, at any given rotational cycle, the predominantly TSI-induced (e.g., sunspot-induced) variability of the upper Balmer activity indices may be de-correlated from the chromospherically sensitive metrics (e.g., the facular area), pending a favorable combination of the two factors - the relative locations of the facular fields and the sunspot groups on the visible solar hemisphere as well as the interplay between magnitudes of the facular-filling and sunspot-filling areas. Both factors are clearly at play in the TOPOMI data (Fig. 2), which span an epoch of low solar activity with few, if any, sunspot groups and sparse facular coverage that is frequently decoupled from the sunspot maxima. Such sparsity and decoupling may lead to the observed time-dependent and generally low correlation of the Balmer line and the $\mathrm{CH} 430 \mathrm{~nm}$ line (i.e., Ca II H\&K) activity indices seen in the TROPOMI data. This trend also persists through the epochs of higher solar activity sampled by OMI, providing there is enough spatial and temporal decoupling between the sunspots and faculae.

Unfortunately, the OMI and TROPOMI observations do not cover the spectral region of the $\mathrm{H} \alpha$ line, which is important for numerous solar and astrophysical applications. We leave the discussion of the complex $\mathrm{H} \alpha$ behavior to a future paper.

The cores of the upper Balmer lines are known to probe the upper layers of the solar photosphere and reach into the chromosphere (Avrett \& Loeser 2008). Therefore, the fact that their variability more closely resembles TSI rather than changes in chromospheric indices is surprising. Attempting to reproduce the behavior of the Balmer activity indices, and thus validate our conclusion that the Balmer indices are "sunspot dominated" at rotational temporal scales, we constructed a "toy" model.
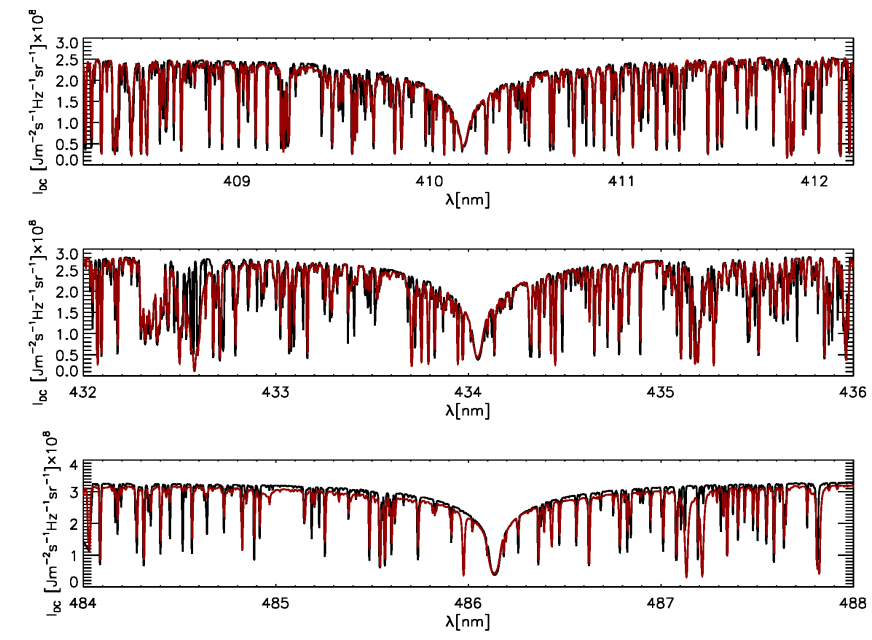

Fig. 4. Comparison of the synthesis of the three Balmer lines obtained with the quiet Sun model at disk center (black lines) with the Hamburg Atlas (red lines) (Neckel 1999).

The approach relies on spectral syntheses produced by 1D static atmosphere models (as published in Fontenla et al. 1999 and expanded in Fontenla et al. 2011) with the Ribicky and Hummer code (RH, Uitenbroek 2001; Kowalski et al. 2017), which is widely used in solar physics applications (Criscuoli et al. 2018; Criscuoli 2019; Berrilli et al. 2020). The synthesis was performed under the assumption of nonlocal thermal equilibrium (NLTE; we note that NLTE treatment is essential for the Balmer series Przybilla \& Butler 2004), using the 20level model atom described in Kowalski et al. (2017). The simulated Balmer-line profiles show good agreement with the solar lines observed at high spectral resolution (Fig. 4) (Neckel 1999); this also applies to the majority of minor transitions seen in the Balmer-line flanks. One may notice some discrepancies in the nearby continua, which, in turn, result from uncertainties in atomic and molecular parameters (Jofré et al. 2014). Indeed, the agreement between the current synthesis and the observations is similar to the agreement achieved with other radiative transfer codes (e.g., ATLAS; Cowley \& Castelli 2002).

The modeled line profiles were convolved with the TROPOMI spectral instrument transfer function. In Fig. 5, we plot TROPOMI observations acquired on March 27, 2019 (the period of very low solar activity), along with the quiet-Sun model profiles. Overall, shapes of the observed Balmer line cores are closely matched by the model. There are some deviations in the line flanks related to the numbers and strengths of minor spectral transitions that are accounted for by the model. Under the OMI and TROPOMI spectral resolutions, these transitions merge and form a pseudo-continuum that may also participate in the solar activity cycles (Mitchell \& Livingston 1991). As defined in this study, the solar activity indices differentiate between the activity in the line cores and the line flanks. This may bias estimates of the line-core activity in the case of the active flanks. However, in the region of interest, $\lambda>400 \mathrm{~nm}$, such biases are diminished by the rapidly decreasing sensitivity of minor transitions (predominantly of photospheric origin) to the events in the solar chromosphere. The perceived bias drawback is amply compensated for by the gains from the very same differential nature of the line activity indices. The line-wing to line-core differentiation efficiently accounts for instrumental effects, thus resulting in unprecedentedly low noise levels, down to $\sim 50 \mathrm{ppm}$ in the TROPOMI case. In order to check the 

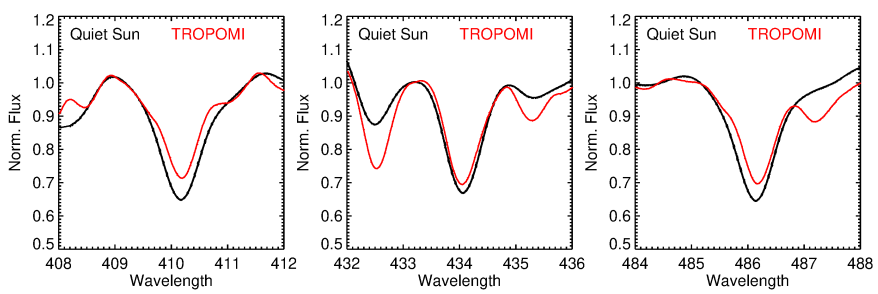

Fig. 5. Quiet Sun model profiles shown in black, and the TROPOMI observations from March 27, 2019, followed by red lines. Both the observations and the models are normalized by the average fluxes sampled at the short-wavelength line flanks (Table 1).

sensitivity of the indices to the choice of the pseudo-continuum regions, we shifted the line-flank domains by $\pm 0.5 \mathrm{~nm}$ in relevance to the ranges listed in Table 1 . This caused slight changes in amplitudes and negligible changes in the forms of the modeled activity cycles.

Constructing the toy model, we used the quiet-Sun spectrum (as above) and assumed that the line cores of Balmer transitions remain unperturbed. This assumption is based on the longterm behavior of Balmer lines in solar cycle 24 (Marchenko $\&$ DeLand 2014). We also assumed that solar flux in the line wings follows the changes in TSI. The model output is compared to observed TROPOMI indices in Fig. 6. Overall, the model reproduces the general observed trends of the first two rotations, which are dominated by the presence of two groups of sunspots.

In the model, the variability is totally ascribed to the change of the continuum intensity as a single variable. We note, however, that the model significantly underestimates the amplitude of the second maximum. It also fails to reproduce the complex shape of the third rotational cycle at $T=2019.43$, which shows pronounced limb effects in the observed Balmer indices. This particular rotation is modulated by the passage of a large decaying region, characterized by the presence of an extensive plage area and two small filaments that lack any observable spots. When the region is at the limbs, the measured Balmer index decreases as the filaments dominate the signal, whereas a small increase is found when the region moves toward the disk center.

Clearly, the proposed single-parameter approach cannot reproduce the contribution of the filaments since the filaments do not significantly modulate TSI (such features appear in the cores of chromospheric lines). The correlation of the modeled Balmer index with the measured index results from the TSI-prescribed changes of the line-wing "continuum" (at OMI/TROPOMI resolution, a blend of minor transitions) that, upon the passage of sunspot groups, depresses the continuum, thus increasing the line activity index. The appearance of extensive facular fields and/or active networks leads to an increase in TSI, which in turn causes the core-to-wing ratio to decrease. The presence of a complex canopy may explain the dimming (the $<1$ values) of the observed index in all three rotations when activity is close to the limb (Fig. 6). This points to complex 3D effects, the reproduction of which is far beyond the scope of the simplified toy model.

\section{Conclusions}

The full-disk solar (Sun-as-a-star) observations reveal complex, dynamic relations between prominent spectral lines involved in the chromospheric activity cycles. Attempting to find the forces driving the changes, we used the near-daily solar observations from OMI and, more recently, TROPOMI and constructed

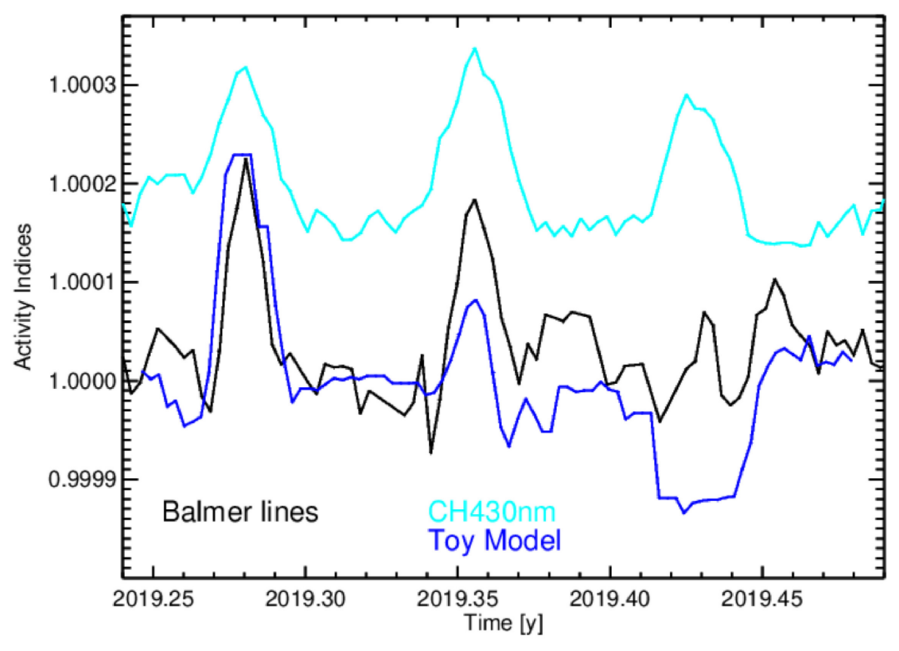

Fig. 6. TROPOMI Balmer activity indices (black line) followed by the "toy" model (blue line). The CH430nm activity indices, which have been normalized, downscaled, and shifted for the sake of clarity, are shown in cyan.

high-quality line activity indices for various spectral transitions and bands. The combination of excellent instrument stability, the near-daily simultaneous acquisition of dozens (OMI) and hundreds (TROPOMI) of solar spectra, and the chosen line activity index approach leads to unprecedentedly low noise levels, $<500 \mathrm{ppm}$ for OMI and $<50 \mathrm{ppm}$ for TROPOMI, which turns out to be crucially important in revealing the cause of variability.

We studied the indices of $\mathrm{H} \beta, \mathrm{H} \gamma$, and $\mathrm{H} \delta$ and find that, on solar rotation timescales, they persistently follow the changes in the TSI, particularly during the passage of sunspots, and thus frequently deviate from the trends seen in the chromospherically sensitive lines and bands, such as $\mathrm{CH} 430 \mathrm{~nm}$, which we used as a reference. This spectral band, as expected, is much less sensitive to photospheric sunspots, however minutely following the changes in the broadly used activity indicator, the CaII H\& K doublet. We stress that these conclusions are primarily driven by the observations.

To interpret the observed trends on rotational timescales, we reconstructed the Balmer index variability by employing a simple model. The model emulates the Balmer index variability by forcing the Balmer line continuum (line wings) to follow the TSI changes that are primarily modulated by sunspot passages.

Contrary to expectations, our study indicates that the upper Balmer line activity indices more closely follow the photospheric variability patterns, compared to chromospheric variability patterns, at rotational timescales. These changes are thus predominantly governed by the appearance and passage of sunspot groups, with relatively smaller variations linked to areas populated by faculae and active networks. We attribute this result to two factors. First, as indicated by Przybilla \& Butler (2004), two out of the three studied Balmer lines $(\mathrm{H} \gamma$ and $\mathrm{H} \delta$ ) mostly arise in the solar photosphere, with the line-core intensities thus largely insensitive to the chromospheric stratification. Second, the modest spectral resolution of OMI/TROPOMI renders the constructed line activity indices less sensitive to the relatively small (for the studied lines) perturbations coming from the active chromosphere.

This study concludes that, for the Sun, the activity patterns detected in prominent spectral transitions may be driven by different sources. This finding may help interpret the wide variety of stellar activity cases observed in the Sun-like stars that 
harbor planetary systems. Indeed, a proper assessment of the stellar chromospheric activity is an important component in the search for extrasolar planets, both for the eclipse-based approach (transients) and the radial-velocity approach (Keplerian orbits). The Sun may serve as a viable template for this challenging analysis.

Acknowledgements. Part of this work was supported by the NASA grant 80HQTR19C0001. The authors are grateful to Dr. J. Harder for providing the PSPT data. The National Solar Observatory is operated by the Association of Universities for Research in Astronomy, Inc. (AURA) under cooperative agreement with the National Science Foundation.

\section{References}

Amarsi, A. M., Nordlander, T., Barklem, P. S., et al. 2018, A\&A, 615, A139 Avrett, E. H., \& Loeser, R. 2008, ApJS, 175, 229

Barklem, P. S., Piskunov, N., \& O'Mara, B. J. 2000, A\&A, 363, 1091

Berrilli, F., Criscuoli, S., Penza, V., \& Lovric, M. 2020, Sol. Phys., 295, 3

Cincunegui, C., Díaz, R. F., \& Mauas, P. J. D. 2007, A\&A, 469, 309

Coddington, O., Lean, J. L., Pilewskie, P., Snow, M., \& Lindholm, D. 2016, Bull. Am. Meteorol. Soc., 97, 1265

Cowley, C. R., \& Castelli, F. 2002, A\&A, 387, 595

Cram, L. E., \& Woods, D. T. 1982, ApJ, 257, 269

Criscuoli, S. 2019, ApJ, 872, 52

Criscuoli, S., Penza, V., Lovric, M., \& Berrilli, F. 2018, ApJ, 865, 22

Dupree, A. K., Avrett, E. H., \& Kurucz, R. L. 2016, ApJ, 821, L7

Flores, M., González, J. F., Jaque Arancibia, M., Buccino, A., \& Saffe, C. 2016 A\&A, 589, A135

Flores, M., González, J. F., Jaque Arancibia, M., et al. 2018, A\&A, 620, A34

Fontenla, J. M., White, O. R., Fox, P. A., Avrett, E. H., \& Kurucz, R. L. 1999, ApJ, 518, 480
Fontenla, J. M., Harder, J., Livingston, W., Snow, M., \& Woods, T. 2011, J. Geophys. Res., 116, D20108

Fuhrmann, K., Axer, M., \& Gehren, T. 1994, A\&A, 285, 585

Gardiner, R. B., Kupka, F., \& Smalley, B. 1999, A\&A, 347, 876

Gomes da Silva, J., Santos, N. C., Boisse, I., Dumusque, X., \& Lovis, C. 2014, A\&A, 566, A66

Jofré, P., Heiter, U., \& Soubiran, C. ARA\&A, 57, 571

Kopp, G., \& Lawrence, G. 2005, Sol. Phys., 230, 91

Kowalski, A. F., Allred, J. C., Uitenbroek, H., et al. 2017, ApJ, 837, 125

Lee, J. N., Cahalan, R. F., \& Wu, D. L. 2016, J. Space Weather Space Clim., 6, 33

Levelt, P. F., van den Oord, G. H. J., Dobber, M. R., et al. 2006, IEEE Trans Geosci. Remote Sens., 44, 1093

Levelt, P. F., Joiner, J., Tamminen, J., et al. 2018, Atmos. Chem. Phys., 18, 5699

Linsky, J. 2017, ARA\&A, 55, 159

Livingston, W., Wallace, L., White, O. C., \& Giampapa, M. S. 2007, ApJ, 657, 1137

Livingston, W., White, O. R., Wallace, L., \& Harvey, J. 2010, Mem. Soc. Astron. It., 81,643

Maldonado, J., Phillips, D. F., Dumusque, X., et al. 2019, A\&A, 627, A118

Marchenko, S. V., \& DeLand, M. T. 2014, ApJ, 789, 117

Meunier, N., \& Delfosse, X. 2009, A\&A, 501, 1103

Mitchell, W. E., Jr, \& Livingston, W. 1991, ApJ, 372, 336

Neckel, H. 1999, Sol. Phys., 184, 421

Pereira, T. M. D., Asplund, M., Collet, R., et al. 2013, A\&A, 554, A118

Pilewskie, P., Kopp, G., Richard, E., et al. 2018, Proc. EGUGA, 5527

Przybilla, N., \& Butler, K. 2004, ApJ, L61

Schenkeveld, V. M. E., Jaross, G., Marchenko, S., et al. 2017, Atmos. Meas. Tech., 10, 1957

Thompson, A. P. G., Watson, C. A., Haywood, R. D., et al. 2020, MNRAS, 494, 4279

Uitenbroek, H. 2001, ApJ, 557, 389

Veefkind, J. P., Aben, I., McMullan, K., et al. 2012, Remote Sens. Environ., 120, 70 